Research Paper

\title{
Clinicopathologic factors affecting discrepancies in HER2 overexpression between core needle biopsy and surgical biopsy in breast cancer patients according to neoadjuvant treatment or not
}

\author{
Young-Hoon Jeong, MD1' Soon Auck Hong, MD., PhD², Hye Shin Ahn, MD., PhD³, Soo kyung Ahn, MD., \\ $\mathrm{PhD}^{4}$, Min Kyoon Kim, MD., PhD ${ }^{\bowtie}$ \\ 1. Department of Surgery, Chung-Ang University College of Medicine, 224-1, Heukseok-dong, Dongjak-gu, Seoul 06973, Republic of Korea. \\ 2. Department of Pathology, Chung-Ang University College of Medicine, 224-1, Heukseok-dong, Dongjak-gu, Seoul 06973, Republic of Korea. \\ 3. Department of Radiology, Chung-Ang University College of Medicine, 224-1, Heukseok-dong, Dongjak-gu, Seoul 06973, Republic of Korea. \\ 4. Department of Surgery, Kangnam Sacred Heart Hospital, Hallym University College of Medicine, 1 Shingil-ro, Youngdeungpo-ku, Seoul. \\ $\triangle$ Corresponding authors: Min Kyoon Kim, MD. Ph. D., Department of Surgery, Chung-Ang University College of Medicine, 221,Heuksuk-dong, Dongjak-gu, Seoul 156- \\ 756, Republic of Korea. TEL: +82-2-6299-1562; E-mail: minkyoon96@cau.ac.kr; likeicetea@hanmail.net; Soo kyung Ahn, MD. Ph. D., Department of Surgery, Kangnam Sacred \\ Heart Hospital, Hallym University College of Medicine, 1 Shingil-ro, Youngdeungpo-ku, Seoul, 07441, Republic of Korea. TEL: 82-2-829-5205; Fax: 82-2-829-5834; E-mail: \\ sooahn79@gmail.com.
}

(C) The author(s). This is an open access article distributed under the terms of the Creative Commons Attribution License (https://creativecommons.org/licenses/by/4.0/). See http://ivyspring.com/terms for full terms and conditions.

Received: 2021.02.15; Accepted: 2021.05.19; Published: 2021.06.04

\begin{abstract}
Background: Accurate determination of human epidermal growth factor receptor 2 (HER2) status on breast core needle biopsy (CNB) tissue is important for determining neoadjuvant chemotherapies (NACs) for primary breast cancer. However, HER2 discrepancies occur before and after surgery, creating difficulties in the administration of appropriate NAC. This study aimed to identify the clinical factors affecting these discrepancies.

Methods: This study was conducted on patients with primary breast cancer who underwent breast surgery from January 2012 to December 2018 at the Chung-Ang University Hospital. HER2 status was analyzed using immunohistochemistry. HER2 was graded as 0 to +3 , and all $2+$ cases were evaluated for gene amplification. The patients were divided into two groups based on whether or not they received chemotherapy. Patient and clinical characteristics between the two groups were compared using the $\chi 2$ test and a logistic regression model.

Results: A total of 443 patients were evaluated; 384 patients (86.7\%) did not receive NAC, and 59 patients (13.3\%) received NAC. The HER2 discordance rate was $12.5 \%$ in the no NAC group and $23.7 \%$ in the NAC group. Most cases showed a change in HER2 status from negative in CNB to positive in surgical biopsy (SB). Clinicopathological factors affecting HER2 change in the no NAC group were larger tumor size and higher histologic grade. Meanwhile, poor response to prior chemotherapy affected HER2 change in NAC.

Conclusion: The overall accuracy of CNB in determining HER2 status was lower in the NAC group than in the no NAC group. Some clinicopathological factors may affect HER2 changes in each group at different levels. Based on the HER2 status at the time of diagnosis, the choice of HER2-targeted therapy may vary, even if this is not true. Future research on the effects of changes in HER2 status between CNB and SB on prognosis is needed.
\end{abstract}

Key words: HER2, breast cancer, tumor heterogeneity, neoadjuvant treatment

\section{Introduction}

Neoadjuvant chemotherapy (NAC) is one of the standard treatments for patients with primary breast cancer [1-3]. With improved treatment results and access to neoadjuvant human epidermal growth factor receptor 2 (HER2)-targeted drugs, a precise preoperative assessment of tumor characteristics has become more important [4-6]. 
CNB is used to confirm HER2 status in the determination of preoperative treatments for primary breast cancer. The HER2/neu gene is amplified in $15-20 \%$ of breast cancers. HER2-targeting agents (e.g., trastuzumab, lapatinib, pertuzumab, trastuzumab emtansine (TDM-1)) are used to treat HER2-positive breast cancer and improve survival [7, 8]. In the neoadjuvant setting, HER2-positive breast cancers with a non-pathologic complete response (non-pCR) have poorer outcomes than those with a pathologic complete response (pCR) [9]. The correct determination of HER2 in $\mathrm{CNB}$ is crucial for improved patient outcomes.

Despite the overall high concordance rate of HER2 status between CNB and surgical biopsy (SB), several cases confuse surgical oncologists when choosing appropriate systemic therapy. If tumors with HER2-negative CNB results become HER2-positive after NAC, we lose the opportunity to improve patient prognosis by achieving a $\mathrm{pCR}$ with prior targeted therapy before surgery. In some other tumors, physicians have reported HER2-positive status on $\mathrm{CNB}$ but negative on SB. These readings may have different interpretations, depending on whether or not the patient received any systemic neoadjuvant treatment. We need to determine the prognostic impact and potential advantages of additional adjuvant therapy with trastuzumab on such patients. However, no study has analyzed HER2 discrepancies between CNB and SB according to prior chemotherapy.

This study examines the discrepancy between CNB and SB for HER2 status and analyzes the clinical factors indicating the differences in situations with or without preoperative chemotherapy.

\section{Methods}

\section{Patients and Ethical Approval}

We retrospectively reviewed the medical records of patients who underwent breast cancer surgery at the Chung-Ang University Hospital from January 2012 to December 2018. A pathology review was performed by a pathologist. We analyzed the clinicopathologic characteristics of patients such as age, body mass index (BMI), menopausal status, type of breast surgery, clinical T, N stage, histologic grade, ER, PgR, HER2 state, and HER2 discrepancies between CNB and SB.

We identified a total of 752 patients initially. We divided the total sample into two groups (with or without preoperative chemotherapy). We excluded patients with unknown NAC, in situ breast cancer, uncertain HER2 status, and those with PCR after NAC because residual tumor for the assessment of biomarkers was unavailable. Additionally, we excluded patients with unknown clinical factor records. A total of 443 patients met the inclusion criteria (Figure 1). This study was approved by the Institutional Review Board of the Chung-Ang University Hospital (IRB No. 2007-036-19326).

\section{Pathological Assessment}

CNB specimens were retrieved from tumor centers by using an ultrasound-guided 14-gauge needle (Table 1$) .348$ patients $(78.6 \%)$ were examined at Chung-Ang university hospital, and 95 patients

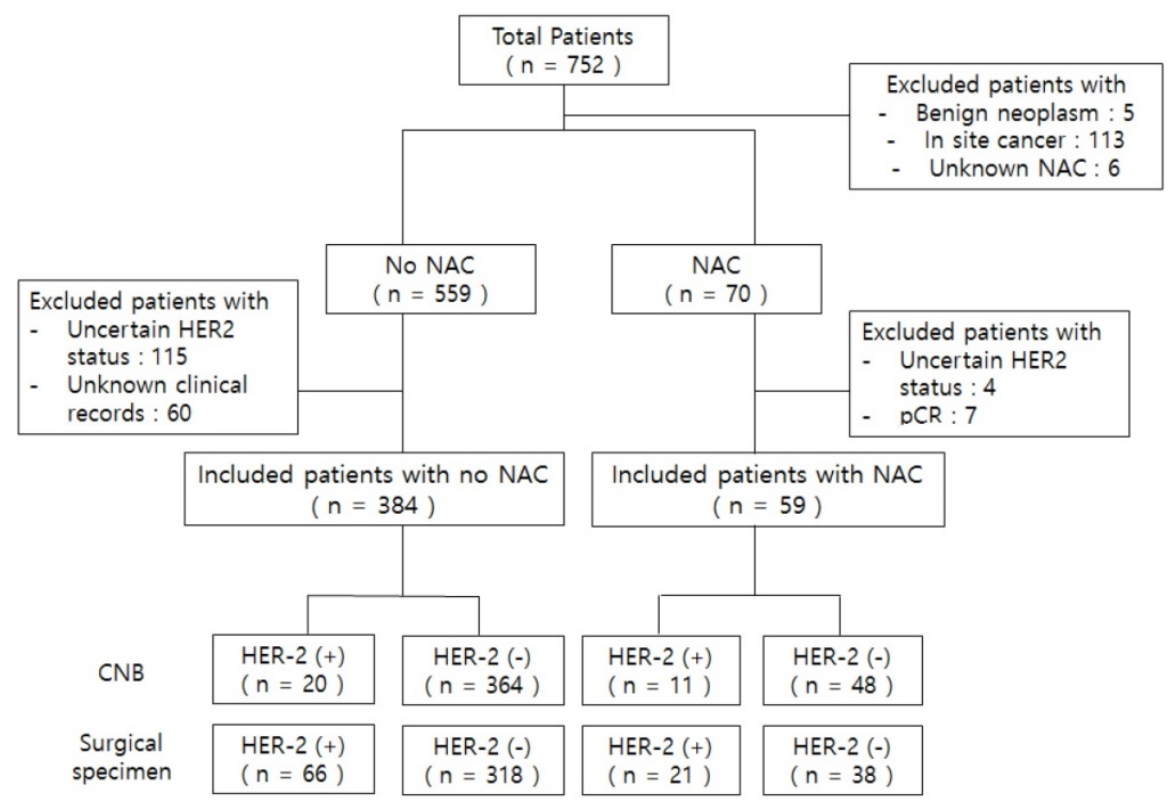

Figure 1. Flow chart of patients who participated in this study. *Uncertain HER2 status: IHC 2+ but no FISH or unknown HER2 status in CNB and surgical specimen. *Abbreviations: NAC, neoadjuvant chemotherapy; $\mathrm{PCR}$, pathologic complete response after neoadjuvant chemotherapy. 
(21.4\%) were examined at another hospital. In 348 patients, 344 patients were examined $\mathrm{CNB}$ and 1 patient was examined vacuum assisted breast biopsy (VABB). Also, 3 patients were examined diagnostic surgical excision. In 95 patients, 87 patients were examined $\mathrm{CNB}$, and 3 patients were examined VABB.

Also 5 patients were examined diagnostic surgical excision. VABB used 11-gauge needle. Patients who underwent $\mathrm{CNB}$ obtained an average of 4.67 specimens. Patients who underwent VABB obtained an average 9.00 specimens. All immunostaining procedures and subsequent interpretations were performed at our institution. Pathology results were interpreted by a senior pathologist with abundant experience. IHC analysis was performed to evaluate the expression of ER, PgR, and HER2 in CNB and surgical specimens. ER and $\mathrm{PgR}$ expression was calculated as the percentage of cells showing a definite nuclear staining status. The cut-off value for ER and PgR positivity was $\geq 1 \%$ of tumor cells positive for nuclear staining.

Table 1. The method of preoperative breast cancer biopsy and average specimen number

\begin{tabular}{llll}
\hline & Biopsy method & $\begin{array}{l}\text { Patient } \\
(\mathrm{N}=443)\end{array}$ & $\begin{array}{l}\text { Specimen } \\
\text { number }\end{array}$ \\
\hline Our Hospital & 14-Gauge Core needle biopsy & $344(77.7)$ & 4.67 \\
& 11-Gauge Vacuum assisted biopsy & $1(0.2)$ & 9.00 \\
& Diagnostic surgical biopsy & $3(0.7)$ & \\
Another Hospital & 14-Gauge Core needle biopsy & $87(19.6)$ & \\
& 11-Gauge Vacuum assisted biopsy & $3(0.7)$ & \\
& Diagnostic surgical biopsy & $5(1.1)$ & \\
\hline
\end{tabular}

HER2 status was also assessed in CNB and surgical specimens. HER2 status was scored as 0 to $3+$ by IHC, according to the ASCO/CAP testing guidelines [10]. For HER2, membranous staining was graded as negative (score 0 or $1+$ ), weakly positive (score $2+$ ), and strongly positive (score $3+$ ). In weakly positive cases, HER2 gene amplification was performed. Cases were considered HER2-positive when the IHC score was 3+, or HER2 gene amplification was identified by fluorescence in site hybridization (FISH).

\section{Statistical analysis}

The patient and clinical characteristics of the two groups were compared using the $\chi^{2}$ test. We calculated the accuracy of CNB compared to SB. We investigated whether there was a difference in HER2 discrepancy according to the presence of prior chemotherapy. Logistic regression models were used to analyze the patient and clinical characteristics affecting the change in HER2 status. P-values less than 0.05 were considered to indicate statistical significance. All statistical analyses were performed using SPSS version 20.0 software.

Table 2. Demographics of Included Patients $(\mathrm{N}=498)$

\begin{tabular}{|c|c|c|c|}
\hline & No NAC $(\mathrm{N}=384)$ & NAC $(\mathrm{N}=59)$ & P-value \\
\hline \multicolumn{4}{|l|}{ Age } \\
\hline Mean age, years & 55.2 & 49.2 & \\
\hline Range & $44.2-66.2$ & 39.3-59.1 & \\
\hline \multicolumn{4}{|l|}{ Body mass index } \\
\hline Mean, $\mathrm{kg} / \mathrm{m}^{2}$ & 24.36 & 24.56 & \\
\hline Range & 20.41-28.31 & $20.00-29.12$ & \\
\hline \multicolumn{4}{|l|}{ Menopause, n (\%) } \\
\hline Pre-menopausal & $156(40.6)$ & $33(55.9)$ & 0.027 \\
\hline Post-menopausal & $228(59.4)$ & $26(44.1)$ & \\
\hline \multicolumn{4}{|l|}{ Breast surgery type, n (\%) } \\
\hline Total mastectomy & $60(15.6)$ & $26(44.1)$ & $<0.001$ \\
\hline Breast conserving surgery & $324(84.4)$ & $33(55.9)$ & \\
\hline \multicolumn{4}{|l|}{ Axillary surgery type, $\mathrm{n}(\%)$} \\
\hline Axillary lymph node dissection & $108(28.1)$ & $42(71.2)$ & $<0.001$ \\
\hline Sentinel lymph node biopsy & $276(71.9)$ & $17(28.8)$ & \\
\hline \multicolumn{4}{|l|}{ Histology, n (\%) } \\
\hline Invasive ductal carcinoma (IDC) & $341(88.8)$ & $53(89.8)$ & 0.910 \\
\hline Invasive lobular carcinoma (ILC) & $18(4.7)$ & $3(5.1)$ & \\
\hline Others & $25(6.5)$ & $3(5.1)$ & \\
\hline \multicolumn{4}{|l|}{ Tumor size } \\
\hline Mean, mm & 20.2 & 26.6 & \\
\hline Range & $7.1-33.3$ & $8.8-44.4$ & \\
\hline \multicolumn{4}{|l|}{ Clinical T stage, n (\%) } \\
\hline $\mathrm{T} 0, \mathrm{~T} 1$ & $237(61.7)$ & $29(49.1)$ & 0.103 \\
\hline $\mathrm{T} 2, \mathrm{~T} 3, \mathrm{~T} 4$ & $147(38.3)$ & $30(50.9)$ & \\
\hline \multicolumn{4}{|l|}{ Clinical N stage, $\mathbf{n}(\%)$} \\
\hline No & $248(64.6)$ & $28(47.5)$ & 0.011 \\
\hline $\mathrm{N}+$ & $136(35.4)$ & $31(52.5)$ & \\
\hline \multicolumn{4}{|l|}{ Histologic grade, $\mathrm{n}(\%)$} \\
\hline 1 & $70(18.2)$ & $6(10.2)$ & $<0.001$ \\
\hline 2 & $207(53.9)$ & $11(18.6)$ & \\
\hline 3 & $107(27.9)$ & $42(71.2)$ & \\
\hline \multicolumn{4}{|l|}{ ER status, $n(\%)$} \\
\hline Positive & $293(76.3)$ & $34(57.6)$ & 0.002 \\
\hline Negative & $91(23.7)$ & $25(42.4)$ & \\
\hline \multicolumn{4}{|l|}{ PgR status, n (\%) } \\
\hline Positive & $274(71.4)$ & $26(44.1)$ & $<0.001$ \\
\hline Negative & $110(28.6)$ & $33(55.9)$ & \\
\hline \multicolumn{4}{|c|}{ HER2 status in core needle biopsy, $n(\%)$} \\
\hline Positive & $20(5.2)$ & $11(18.6)$ & $<0.001$ \\
\hline Negative & $364(94.8)$ & $48(81.4)$ & \\
\hline \multicolumn{4}{|c|}{ HER2 status in surgical specimen, $\mathrm{n}(\%)$} \\
\hline Positive & $66(17.2)$ & $21(35.6)$ & $<0.001$ \\
\hline Negative & $318(82.8)$ & $38(64.4)$ & \\
\hline
\end{tabular}

\section{Results}

\section{Patient Characteristics According to NAC}

A total of 443 patients were included in the study (Table 2). There were 59 patients (13.3\%) with NAC and 384 patients $(86.7 \%)$ without NAC; $80.5 \%$ of patients underwent breast-conserving surgery, and $19.5 \%$ underwent a total mastectomy. We performed axillary lymph node resection in $33.9 \%$ and sentinel lymph node biopsy in $66.1 \%$ of patients. The median age was younger in the NAC group (55.2 \pm 11.0 years in the no NAC group and 49.2 \pm 9.9 years in the NAC group). The BMIs between the two groups were similar (24.36 in the no NAC group and 24.56 in the NAC group). The majority of pathologic types were invasive ductal carcinoma $(89.0 \%)$, followed by 
invasive lobular carcinoma (4.8\%) and other subtypes $(6.2 \%)$.

Mean tumor size was smaller in the no NAC group (20.2 $\mathrm{mm}$ in the no NAC group, $26.6 \mathrm{~mm}$ in the NAC group). There were 266 patients $(60.0 \%)$ in clinical $\mathrm{T}$ stages 0 or 1 and 177 patients $(40.0 \%)$ in clinical stage 2 or higher. There were 274 patients $(62.3 \%)$ in clinical $\mathrm{N}$ stage 0 and 167 patients (37.7\%) in clinical $\mathrm{N}$ stage 1 or higher. ER positivity rate in SB was $293(66.1 \%)$ in the no NAC group and 34 (7.7\%) in the NAC group. The PgR positivity rate in SB was 274 $(61.9 \%)$ in the no NAC group and $26(5.9 \%)$ in the NAC group.

\section{Concordance of HER2 status between CNB and surgical specimens}

The overall accuracy of $\mathrm{CNB}$ in determining HER2 status was lower in the NAC group than in the no NAC group (Table 3). In 384 patients who did not receive NAC, HER2 status was concordant between needle-biopsied and surgical specimens in 336 patients $(87.5 \%)$. Forty-seven (12.2\%) HER2-negative tumors changed to HER2-positive, while one $(0.3 \%)$ HER2-positive tumor changed to HER2-negative. In 59 patients who received NAC, the HER2 concordance rate was $76.3 \%$, and the HER2 discordance rate was higher than that for no NAC patients (23.7\%). Twelve (20.3\%) HER2-negative tumors changed to HER2-positive, while two (3.4\%) HER2-positive tumors changed to HER2-negative. The HER2 concordance rate according to NAC showed a statistically significant difference ( $p$-value $=0.023$ ). Most cases of HER2 discordance turned to HER2 overexpression in SB. The NAC group had eight patients using trastuzumab, which was positive for HER2 $3+$ in preoperative biopsies. Among them, there were two patients whose HER2 results were negative in surgical specimen. The other six still had positive HER2 results after surgery. However, more patients had no HER2-expression after NAC than the no NAC patients ( $\mathrm{p}$-value $=0.046$ ). Since trastuzumab was used in the previous chemotherapy group, the rate of HER2 status change from positive to negative would have been higher.

Table 3. Accuracy of core needle biopsy compared to surgical specimens

\begin{tabular}{llllllll}
\hline & & \multicolumn{3}{c}{ No NAC } & \multicolumn{3}{c}{ NAC } \\
\cline { 3 - 8 } & & $\mathrm{N}$ & $\%$ & $\begin{array}{l}\text { Concordance } \\
\text { rate }\end{array}$ & $\mathrm{N}$ & $\%$ & $\begin{array}{l}\text { Concordance } \\
\text { rate }\end{array}$ \\
\hline cHER2(-) & sHER2(-) & 317 & 82.6 & 87.5 & 36 & 61.0 & 76.3 \\
cHER2(+) & sHER2(+) & 19 & 4.9 & & 9 & 15.3 & \\
cHER2(+) & sHER2(-) & 1 & 0.3 & & 2 & 3.4 & \\
cHER2(-) & sHER2(+) & 47 & 12.2 & 12 & 20.3 & \\
& Thtal & 384 & 100 & & 59 & 100 & \\
& & & & & & & \\
\hline
\end{tabular}

\section{Clinicopathologic factors associated with HER2 change}

Next, we analyzed the clinicopathological factors affecting HER2 discrepancies. In the no NAC group, menopausal status, tumor size over $2 \mathrm{~cm}$, a higher histologic grade, and ER/PgR negativity were found to affect HER2 discrepancy in univariate analysis (Table 4). Multivariate analysis showed that tumor size (OR 2.518, p-value 0.012) and histological grade (OR-2.182, p-value 0.029) affected HER2 discrepancy (Table 4). Pathologic findings indicated that tumor heterogeneity according to tumor size and the histological grade was related to HER2 discrepancy (Figure 2).

In the NAC group, large residual tumors (yp T $>1$ $\mathrm{cm}$ ) and the status of metastatic lymph nodes (yp $\mathrm{N} \geq 1$ ) affected HER2 discrepancy (Table 4). However, multivariate analysis showed that no factor affected HER2 discrepancy in the NAC group (Table 5).

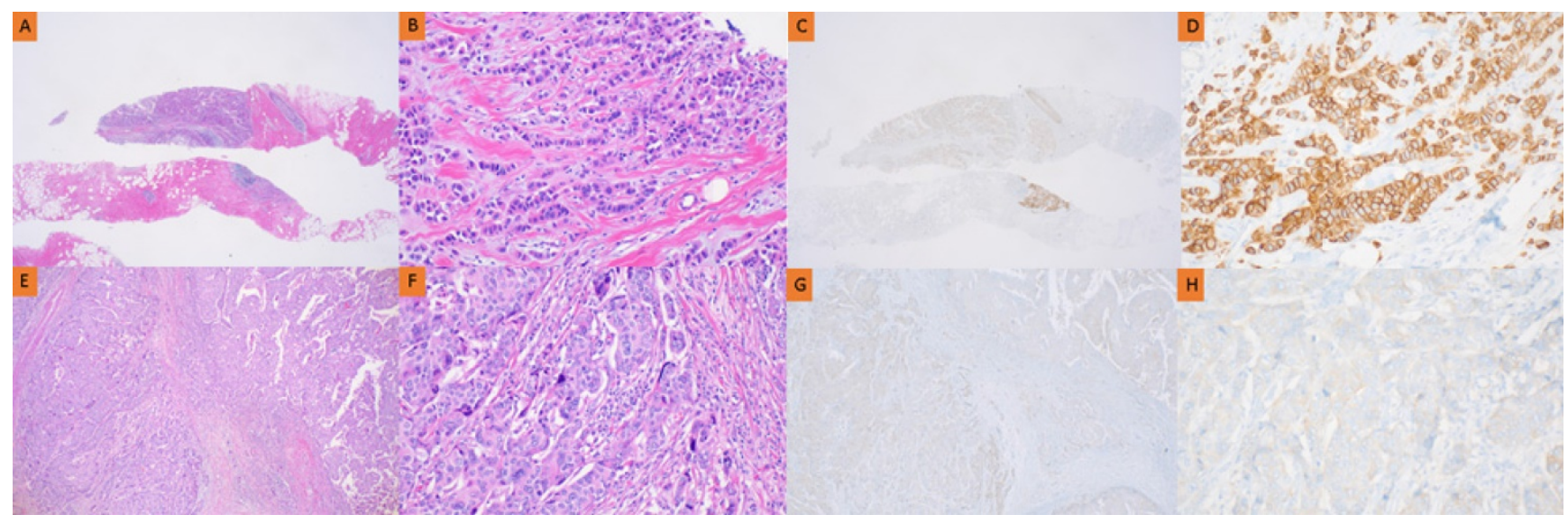

Figure 2. Pathology of HER2 discrepancy between CNB and SB in the no NAC group. Pathology of invasive ductal breast carcinoma in CNB (A, B, C, D) and SB (E, $F, G, H)$. HER2 immunostaining showed strong complete membrane staining in $>10 \%$ of tumor cells (C, D). However, HER2 immunostaining showed faint perceptible membrane staining in $>10 \%$ of tumor cells $(\mathrm{G}, \mathrm{H})$. 
Table 4. Predictive factors associated with HER2 discrepancy in the No NAC group

\begin{tabular}{|c|c|c|c|c|c|c|}
\hline & \multicolumn{3}{|l|}{ Univariate } & \multicolumn{3}{|c|}{ Multivariate } \\
\hline & Concordance & Discordance & $\begin{array}{l}\mathrm{P} \text { - } \\
\text { value }\end{array}$ & OR & $95 \% \mathrm{CI}$ & $\begin{array}{l}\mathrm{P}- \\
\text { value }\end{array}$ \\
\hline Age & & & & 1.502 & $0.469-4.817$ & 0.494 \\
\hline$\leq 40 \mathrm{yrs}$ & $30(7.7)$ & $2(4.2)$ & 0.237 & & & \\
\hline$>40 \mathrm{yrs}$ & $361(92.3)$ & $46(95.8)$ & & & & \\
\hline \multicolumn{7}{|l|}{ Body mass index } \\
\hline$<25 \mathrm{~kg} / \mathrm{m}^{2}$ & $209(62.2)$ & $33(68.8)$ & 0.379 & & & \\
\hline$\geq 25 \mathrm{~kg} / \mathrm{m}^{2}$ & $127(37.8)$ & 15 (31.2) & & & & \\
\hline Menopause, $\mathrm{n}(\%)$ & & & & 2.450 & $0.738-8.134$ & 0.143 \\
\hline Pre-menopausal & $143(37.2)$ & $13(27.1)$ & 0.041 & & & \\
\hline Post-menopausal & $193(50.3)$ & $35(72.9)$ & & & & \\
\hline \multicolumn{7}{|l|}{ Histology, n (\%) } \\
\hline $\begin{array}{l}\text { Invasive ductal } \\
\text { carcinoma (IDC) }\end{array}$ & $297(77.3)$ & 44 (91.6) & 0.761 & & & \\
\hline $\begin{array}{l}\text { Invasive lobular } \\
\text { carcinoma (ILC) }\end{array}$ & $16(4.2)$ & $2(4.2)$ & & & & \\
\hline Others & $23(6.0)$ & $2(4.2)$ & & & & \\
\hline T stage, $\mathbf{n}(\%)$ & & & & 2.518 & $1.228-5.165$ & 0.012 \\
\hline$\leq 2 \mathrm{~cm}$ & $217(56.5)$ & $20(41.7)$ & 0.002 & & & \\
\hline$>2 \mathrm{~cm}$ & $119(31.0)$ & $28(58.3)$ & & & & \\
\hline N stage, n (\%) & & & & 0.411 & $0.193-0.874$ & 0.021 \\
\hline No & $213(55.5)$ & 35 (72.9) & 0.197 & & & \\
\hline $\mathrm{N}+$ & $123(32.0)$ & $13(27.1)$ & & & & \\
\hline \multicolumn{3}{|c|}{ Histologic grade, $\mathrm{n}(\%)$} & & 2.182 & $1.083-4.393$ & 0.029 \\
\hline Low (I-II) & $253(75.3)$ & $24(50.0)$ & $<0.001$ & & & \\
\hline High (III) & $83(24.7)$ & $24(50.0)$ & & & & \\
\hline ER status, n (\%) & & & & 2.993 & $0.970-9.232$ & 0.056 \\
\hline Positive & $273(71.1)$ & $26(54.2)$ & $<0.001$ & & & \\
\hline Negative & $63(16.4)$ & $22(45.8)$ & & & & \\
\hline PgR status, n (\%) & & & & 0.927 & $0.302-2.842$ & 0.894 \\
\hline Positive & $251(65.4)$ & $22(45.8)$ & $<0.001$ & & & \\
\hline Negative & $85(22.1)$ & $26(54.2)$ & & & & \\
\hline
\end{tabular}

\section{Discussion}

Several studies have evaluated the effects of HER2-targeted NAC. The NeoSphere trial [11] suggested that neoadjuvant pertuzumab is beneficial when combined with trastuzumab and docetaxel in progression- and disease-free survival at five years. The TRYPHAENA trial [12] showed that neoadjuvant pertuzumab and trastuzumab given concurrently or sequentially with an anthracycline-and carboplatinbased chemotherapy regimen resulted in an improved pCR rate. With these positive results, the National Health Insurance Service in Korea has started covering the cost of pertuzumab for neoadjuvant HER2-positive breast cancer patients. Furthermore, accurate determination of HER2 status before surgery has become crucial.

Reports show that the concordance rate (CR) of the biomarker status between $\mathrm{CNB}$ and surgical specimens is $79-100 \%$ [13]. In a recent analysis, the CR of surgical specimens with CNBs for hormone receptor status had a $95.1 \%$ agreement. However, the CR of HER2 IHC was lower than that at 81.4\% [14], similar to previous reports on HER2 discrepancy (ranging from 61-97.3\%) [15]. We treated the NAC or no NAC groups separately, and our results showed a higher rate $(87.5 \%)$ of HER2 concordance in the no NAC group. The NAC group showed a higher rate ( $23.7 \%$ vs. $12.5 \%)$ of HER2 discordance.

Table 5. Predictive factors associated with HER2 discrepancy in the NAC group

\begin{tabular}{|c|c|c|c|c|c|c|}
\hline & \multicolumn{3}{|l|}{ Univariate } & \multicolumn{3}{|c|}{ Multivariate } \\
\hline & Concordance & Discordance & $\begin{array}{l}\mathrm{P} \text { - } \\
\text { value }\end{array}$ & OR & $95 \% \mathrm{CI}$ & $\begin{array}{l}\mathrm{P} \text { - } \\
\text { value }\end{array}$ \\
\hline \multicolumn{7}{|l|}{ Age } \\
\hline$\leq 40$ yrs & $10(22.2)$ & $1(7.1)$ & 0.196 & & & \\
\hline$>40 \mathrm{yrs}$ & $35(77.8)$ & $13(92.9)$ & & & & \\
\hline \multicolumn{7}{|l|}{ Body mass index } \\
\hline$<25 \mathrm{~kg} / \mathrm{m}^{2}$ & $30(50.8)$ & $8(13.6)$ & 0.516 & & & \\
\hline$\geq 25 \mathrm{~kg} / \mathrm{m}^{2}$ & $15(25.4)$ & $6(10.2)$ & & & & \\
\hline \multicolumn{7}{|c|}{ Menopause, n (\%) } \\
\hline Pre-menopausal & $27(45.8)$ & $6(10.2)$ & 0.259 & & & \\
\hline Post-menopausal & $18(30.5)$ & $8(13.6)$ & & & & \\
\hline \multicolumn{7}{|l|}{ Histology, n (\%) } \\
\hline $\begin{array}{l}\text { Invasive ductal } \\
\text { carcinoma (IDC) }\end{array}$ & $39(66.1)$ & $14(23.7)$ & 0.354 & & & \\
\hline $\begin{array}{l}\text { Invasive lobular } \\
\text { carcinoma (ILC) }\end{array}$ & $3(5.1)$ & $0(0.0)$ & & & & \\
\hline Others & $3(5.1)$ & $0(0.0)$ & & & & \\
\hline ypT stage, n (\%) & & & & 2.981 & $0.65-13.75$ & 0.161 \\
\hline$\leq 1 \mathrm{~cm}$ & $8(17.8)$ & $6(42.9)$ & 0.051 & & & \\
\hline$>1 \mathrm{~cm}$ & $37(82.2)$ & $8(57.2)$ & & & & \\
\hline ypN stage, n ( $\%)$ & & & & 1.720 & $0.37-7.93$ & 0.487 \\
\hline No & $18(30.5)$ & $10(16.9)$ & 0.040 & & & \\
\hline $\mathrm{N}+$ & $27(45.8)$ & $4(6.8)$ & & & & \\
\hline \multicolumn{7}{|c|}{ Histologic grade, n (\%) } \\
\hline Low (I-II) & $15(33.3)$ & $2(14.3)$ & 0.273 & & & \\
\hline High (III) & $30(66.7)$ & $12(85.7)$ & & & & \\
\hline \multicolumn{7}{|l|}{ ER status, $n(\%)$} \\
\hline Positive & $24(40.7)$ & $9(15.3)$ & 0.471 & & & \\
\hline Negative & $21(35.6)$ & $5(8.5)$ & & & & \\
\hline \multicolumn{7}{|l|}{ PgR status, n (\%) } \\
\hline Positive & $20(33.9)$ & $6(10.2)$ & 0.917 & & & \\
\hline Negative & $25(42.4)$ & $8(13.6)$ & & & & \\
\hline
\end{tabular}

The observed changes in HER2 status from positive to negative are rare. However, this phenomenon occurs more often in NAC cases and is observed more frequently with statistical significance, probably due to neoadjuvant HER2-targeted treatments. Possible underlying mechanisms include targeted therapies for chemo-sensitive tumor cells, which leave behind insensitive tumor cells with different biology in the residual tumor [16]. Otherwise, changes in receptor expression of cells provide a survival mechanism for tumor cells, leading to cytotoxic drug resistance. Additionally, the expression of ER, PR, and HER2 are highly inter-dependent, and changing one receptor with NAC can modulate other receptors. Therefore, chemotherapy-induced hypoestrogenism may change HER2 status and estrogen and progesterone receptor expression [17].

Similar results were reported by a recent Japanese study showing that the rate of transition from HER2-positive to negative was three times greater after NAC [18]. They evaluated the prognostic impact of changes in HER2 status in patients with 
HER2-positive tumors and found no difference in disease-free survival between patients with and without changes in HER2 status after NAC. Two other retrospective studies assessed the prognostic impact of changes in HER2 status after NAC in patients with primary breast cancer. Among patients with residual disease after NAC, Mittendorf et al. (25 patients) and Guarneri et al. (69 patients) demonstrated that patients with HER2 loss tended to have a higher risk of recurrence than those who maintained HER2 positivity $[19,20]$. Selective pressure on resistant HER2-negative clones and the induction of HER2 loss were the expected mechanisms of resistance and a reason for a poorer prognosis.

This study showed that the predictive factors affecting HER2 discrepancy in the no NAC group were related to menopause, breast surgery type, histologic grade, tumor size exceeding $2 \mathrm{~cm}$, and $\mathrm{ER} / \mathrm{PgR}$ negativity. Breast cancer is known as a heterogeneous disease with intertumoral and intratumoral heterogeneity. Cancer stem cells and their clonal evolution model could describe intra-tumor heterogeneity, and the tumor microenvironment could contribute to the complexity of these tumors. Structural pathological information on how well tubules form has been thought to lead to discordance between CNB and SB.

What we found in our study is that, rather than the change of HER2 to negative by treatment, the limitation of core biopsies, the tumor as a whole, and the heterogenicity of breast cancer itself, HER2 is often positively changed in surgical tissue. To reduce the discrepancy between $\mathrm{CNB}$ and surgical specimens, patients with the above-mentioned clinical factors need to undergo multiple CNB if possible. For accurate histologic diagnosis of breast cancer, at least four cores should be obtained using a 14-gauge needle [21].

Our study has some limitations. The case number was small, especially for NAC cases. Neoadjuvant HER2-targeted treatments were performed with trastuzumab only for almost all patients included in this study. In South Korea, pertuzumab has been covered by the National Health Insurance since 2019. Furthermore, we did not confirm the effects of HER2 status discrepancy between $\mathrm{CNB}$ and SB on patient prognosis. However, if a surgical oncologist experiences HER2 discrepancy between CNB and SB, our CNB's HER2 prediction tendency and clinically influential factors will help clinicians avoid confusion in deciding further treatment.

\section{Conclusion}

The HER2 discordance rate was higher in the group with NAC (23.7\%) than in the group without NAC (12.5\%). Most HER2 discordance cases were HER2-negative in CNB but confirmed as HER2positive in the surgical specimen. Clinicopathological factors affecting HER2 changes in the no NAC group were large tumor size $(>2 \mathrm{~cm})$ and high histologic grade. A large residual tumor (yp $\mathrm{T}>1 \mathrm{~cm}$ ) and lymph node metastasis status (yp $\mathrm{N} \geq 1$ ) had marginal effects on HER2 discrepancy in the NAC group. Future studies on the survival rate and prognosis according to these clinicopathological factors are necessary.

\section{Acknowledgements}

This research was supported by the Chung-Ang University Research Grants in 2020, and National Research Foundation of Korea (NRF) grant funded by the Korea government (MSIT) (No. 20201452).

\section{Competing Interests}

The authors have declared that no competing interest exists.

\section{References}

1. Fisher B, Brown A Fau - Mamounas E, Mamounas E Fau - Wieand S, et al. Effect of preoperative chemotherapy on local-regional disease in women with operable breast cancer: findings from National Surgical Adjuvant Breast and Bowel Project B-18. J Clin Oncol. 1997; 15(7):2483-93.

2. Fisher B, Bryant J Fau - Wolmark N, Wolmark N Fau - Mamounas E, et al. Effect of preoperative chemotherapy on the outcome of women with operable breast cancer. J Clin Oncol. 1998; 156(8):2672-85.

3. Wolmark N, Wang J Fau - Mamounas E, Mamounas E Fau - Bryant J, Bryant J Fau - Fisher B, Fisher B. Preoperative chemotherapy in patients with operable breast cancer: nine-year results from National Surgical Adjuvant Breast and Bowel Project B-18. J Natl Cancer Inst Monogr. 2001; 30:96-102.

4. Rouzier R, Perou Cm Fau - Symmans WF, Symmans Wf Fau - Ibrahim N, et al. Breast cancer molecular subtypes respond differently to preoperative chemotherapy. Clin Cancer Res. 2005; 11(16):5678-85.

5. Carey LA, Dees Ec Fau - Sawyer L, Sawyer L Fau - Gatti L, et al. The triple negative paradox: primary tumor chemosensitivity of breast cancer subtypes. Clin Cancer Res. 2007; 13(8):2329-34.

6. Esserman LJ, Berry Da Fau - Cheang MCU, Cheang Mc Fau - Yau C, et al. Chemotherapy response and recurrence-free survival in neoadjuvant breast cancer depends on biomarker profiles: results from the I-SPY 1 TRIAL (CALGB 150007/150012; ACRIN 6657). (1573-7217 (Electronic)). Breast Cancer Res. Treat. 2012; 132(3):1049-1062.

7. Slamon DJ, Leyland-Jones B, Shak S, et al. Use of chemotherapy plus a monoclonal antibody against HER2 for metastatic breast cancer that overexpresses HER2. N Engl J Med. 2001;344(11):783-792.

8. Piccart-Gebhart MJ, Procter M, Leyland-Jones B, et al. Trastuzumab after adjuvant chemotherapy in HER2-positive breast cancer. $N$ Engl I Med. 2005;353(16):1659-1672.

9. von Minckwitz G, Untch M, Blohmer JU, et al. Definition and impact of pathologic complete response on prognosis after neoadjuvant chemotherapy in various intrinsic breast cancer subtypes. J Clin Oncol. 2012;30(15):1796-1804.

10. Wolff AC, Hammond MEH, Allison KH, et al. Human Epidermal Growth Factor Receptor 2 Testing in Breast Cancer: American Society of Clinical Oncology/College of American Pathologists Clinical Practice Guideline Focused Update.Arch Pahol Lab Med. 2018; 142(11):1364-1382.

11. Gianni L, Pienkowski T, Im YH, et al. 5-year analysis of neoadjuvant pertuzumab and trastuzumab in patients with locally advanced, inflammatory, or early-stage HER2-positive breast cancer (NeoSphere): a multicentre, open-label, phase 2 randomised trial. (1474-5488 (Electronic)). Lancet Oncol. 2016; 17(6):791-800.

12. Schneeweiss A, Chia S Fau - Hickish T, Hickish T Fau - Harvey V, et al. Pertuzumab plus trastuzumab in combination with standard neoadjuvant anthracycline-containing and anthracycline-free chemotherapy regimens in patients with HER2-positive early breast cancer: a randomized phase II cardiac safety study (TRYPHAENA). Ann Oncol. 2013; 24(9):2278-84.

13. Meattini I, Bicchierai G, Saieva C, et al. Impact of molecular subtypes classification concordance between preoperative core needle biopsy and surgical specimen on early breast cancer management: Single-institution 
experience and review of published literature. Eur J Surg Oncol. 2017;43(4):642-648.

14. Jeong YS, Kang J, Lee J, Yoo TK, Kim SH, Lee A. Analysis of the molecular subtypes of preoperative core needle biopsy and surgical specimens in invasive breast cancer. J Pathol Transl Med. 2020;54(1):87-94.

15. Jeong YS, Kang J, Lee J, Yoo TK, Kim SH, Lee A. Analysis of the molecular subtypes of preoperative core needle biopsy and surgical specimens in invasive breast cancer. J Pathol Transl Med. 2020; 54(1): 87-94.

16. van de Ven S, Smit VT, Dekker TJ, Nortier JW, Kroep JR. Discordances in ER, PR and HER2 receptors after neoadjuvant chemotherapy in breast cancer. Cancer Treat Rev. 2011;37(6):422-430.

17. Bines J, Oleske DM, Cobleigh MA. Ovarian function in premenopausal women treated with adjuvant chemotherapy for breast cancer. J Clin Oncol. 1996;14(5):1718-1729.

18. Yoshida A, Hayashi N, Suzuki K, Takimoto M, Nakamura S, Yamauchi H. Change in HER2 status after neoadjuvant chemotherapy and the prognostic impact in patients with primary breast cancer. J Surg Oncol. 2017;116(8):1021-1028

19. Mittendorf EA, Wu Y, Scaltriti M, et al. Loss of HER2 amplification following trastuzumab-based neoadjuvant systemic therapy and survival outcomes. Clin Cancer Res. 2009;15(23):7381-7388.

20. Guarneri V, Dieci MV, Barbieri E, et al. Loss of HER2 positivity and prognosis after neoadjuvant therapy in HER2-positive breast cancer patients. Ann Oncol. 2013;24(12):2990-2994.

21. Fishman JE, Milikowski C Fau - Ramsinghani R, Ramsinghani R Fau Velasquez MV, Velasquez Mv Fau - Aviram G, Aviram G. US-guided core-needle biopsy of the breast: how many specimens are necessary? (0033-8419 (Print)). 